\title{
Treatment of the Anterior Crossbite in Mixed Dentition: Case Reports
}

\section{Mohanad Alsaadi*}

Pediatric Dentistry, University of Greifswald, Germany

*Corresponding Author: Mohanad Alsaadi, Pediatric Dentistry, University of Greifswald, Germany.

DOI: $10.31080 /$ ASDS.2020.04.0987
Received: May 16, 2020

Published: November 19, 2020

(C) All rights are reserved by Mohanad

Alsaadi.

\begin{abstract}
Anterior crossbite is a malocclusion which may resulted in many complications which can be avoided by early diagnosis and intervention with fixed or removable appliances. This study aimed to presenting different results of treating anterior crossbite during mixed dentition in different age of three attended children by using removable acrylic appliance with bite plan and to summarize the advantages and disadvantages of the other non- selected methods in this study. Clinical examination of children aged 8-10 who attended with chief complaint of crowding and esthetically displeasure showed one or two permanent maxillary incisors position behind the permanent lower incisors in each case. After clinical and radiographical examination, removable acrylic appliances with bite plans were selected to treat the three cases. Treatment periods were 6-8 weeks according to the cases severity. The selected appliance had been discussed in comparison with the non-selected methods. The first step to treat the anterior crossbite is to know the indications of each treatment methods in order to apply the suitable one for each case.
\end{abstract}

Keywords: Anterior Crossbite; Acrylic Appliance; Mixed Dentition; Malocclusion; Eruption; Crowding

\section{Introduction}

Anterior crossbite is defined as the malocclusion that results when the position of maxillary anterior teeth being behind the mandibular anterior teeth [1]. It is an esthetic and functional concern to the child and parents during the mixed dentition of a child. The responsibility of the dentist or orthodontist in this condition is to guide the teeth into normal alignment during the stage of oral-facial growth and development. The mixed dentition period represents the greatest opportunity for occlusal guidance and interception of malocclusion. If this condition delayed until the late developmental stage of maturity, the treatment will be more difficult and complicated [2]. Its prevalence in many countries around the world between $2.2 \%$ and $36 \%$ [3]. There are many conditions which were implicated for causing this malocclusion such as supernumerary teeth, odontomas, persistent primary teeth, traumatized primary incisors, habitual lip or nail biting and lack of space in dental arch [4].

Late diagnosis of the anterior crossbite may lead to many complications which were reported in many previous studies. These complications included of tooth wear, anterior tooth fractures, gingival inflammations, temporomandibular joints disorders and malfunction of occlusion and esthetic. Early diagnosis will shorten the treatment period by directing the erupted tooth into the normal position before deteriorating the condition with total eruption of the maxillary anterior teeth behind the lower anterior teeth [5].

The treatments included many methods with different advantages and disadvantages. It can perform by fixed or removable appliances which have to be activated to create forces that move the maxillary teeth facially into the normal position. Since many years, the different treatment methods had been discussed in many previous clinical studies which included tongue blade, reversed stainless steel crowns, composite inclined plane, fixed orthodontic brackets, and removable acrylic appliances with or without bite plane as the mouth most of time is open and not usually the bite plane is needed [6].

In addition to that, many previous studies mentioned that the decision of using a specific method for managing the crossbite condition depends on many factors which included child's age, number of teeth required to be treated, status of occlusion, cooperation of 
the child and the parents, and the clinician qualification and experience [7].

\section{Aim}

The aim of this study is to demonstrate the result of three different cases of anterior crossbite in early and late developmental stages which were managed by using removable active acrylic appliances with bite plane and soldered finger springs or key screw. In addition to that, a summary of the different methods will be mentioned and discussed to clarify the advantages of the selected appliance to correct the malocclusions in this study and the disadvantages of the other appliances which were not selected for these three cases.

\section{Case Reports}

In the pediatric dentistry practice, three children aged 8-10 years were attended with chief complaint of displeasure with appearance of their maxillary anterior teeth. After they were examined, there were two maxillary anterior teeth of each child were positioned behind the permanent lower anterior teeth for two patients (Figure 1a and 2a) while the third patient who aged 8-years had only one maxillary permanent central incisor located behind the lower permanent anterior teeth with insufficient space for the adjacent maxillary permanent lateral incisor (Figure 3a). The mesiodistal spaces were analyzed to confirm the sufficient space for the involved teeth in each case before starting the treatment. In addition to that, facial profiles were examined and there were no skeletal abnormalities for the three attended children. The nails and fingers were examined to detect any sign of habitual biting. A clear bite marks were detected on the dorsum of both hands of one child from the three children which indicate an aggressive habitual biting in these areas (Figure 2d). Moreover, there was no family history of this malocclusion was noticed. Removable active acrylic appliances with soldered finger springs were selected to managing the three cases after informed the parents about the treatment plan with a consent form which was obtained from each parent. Rather than the soldered finger spring, the activation of the applied force to the partially erupted case had been achieved by using a key screw. After the impressions were taken by using irreversible hydrocolloid impression material, alginate, the study models were sent to the laboratory to fabricate the selected appliances. The appliances were placed into the mouths after they were activated and the instruction of cleaning and keeping good oral hygiene as well as the follow-up appointments were given to all parents. The patients and their parents were informed about the importance of using the appliances regularly and to remove them only during meals. Two patients were called once a week for follow-up during the treatment period which was from 6 to 8 weeks. The third whose appliance was activated by a screw lived far away from the practice and his parents were informed to attend after treatment with weekly online contacts (Figure 3a). At the end of this period, all the maxillary anterior teeth were placed in their normal positions labially to the lower anterior permanent teeth (Figure 1b, 2b, 3b).

Case 1

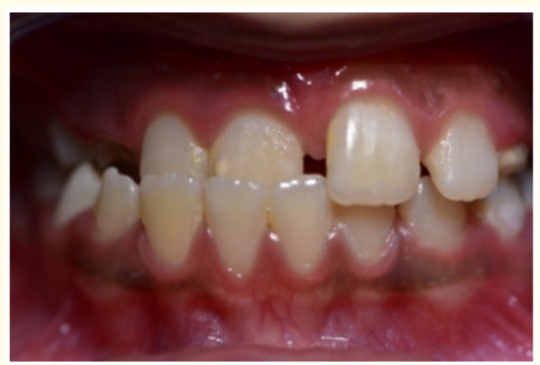

Figure 1a

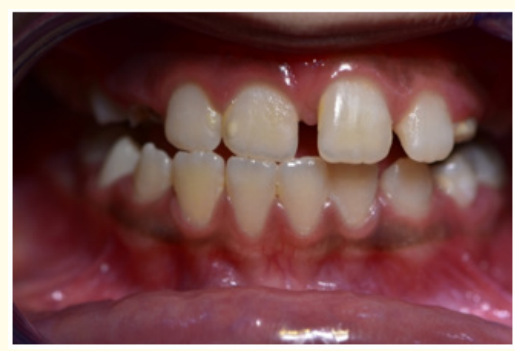

Figure 1b

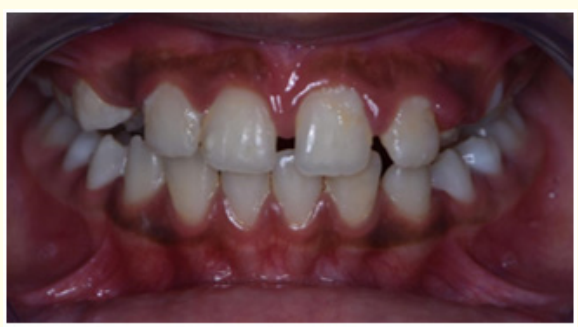

Figure 1c

Figure 1: (a) A 9-year-old male patient with crossbite of 11, 12 teeth numbered. (b) An improvement had been seen after 3 weeks of treatment. (c) Final result after 8 weeks with normal occlusion and position of the maxillary permanent central and lateral incisors. 
Case 2

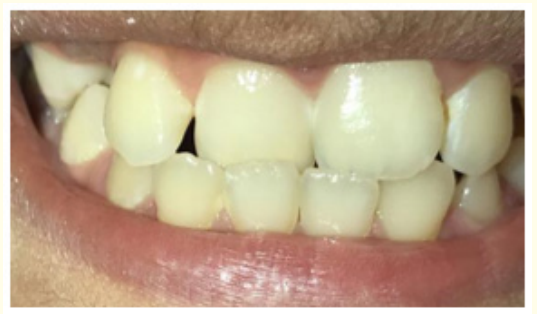

Figure 2a

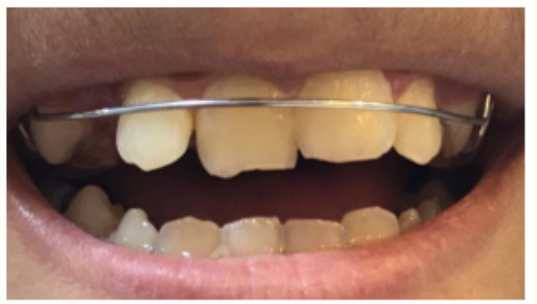

Figure 2b

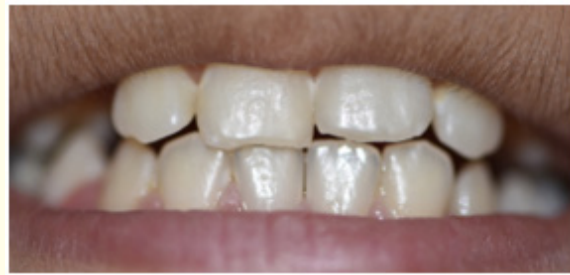

Figure 2c

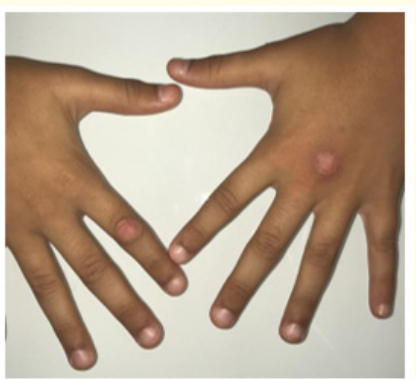

Figure 2d

Figure 2: A 10-year-old male patient with crossbite of \#11. (b) Placement of the selected removable acrylic appliance with bite plan at the beginning of the treatment (c) After 7 weeks, the tooth \#11 migrated out the crossbite. (d) Bite marks were observed on the dorsum of both hands as the result of habitual biting which affected the tooth direction.
Case 3

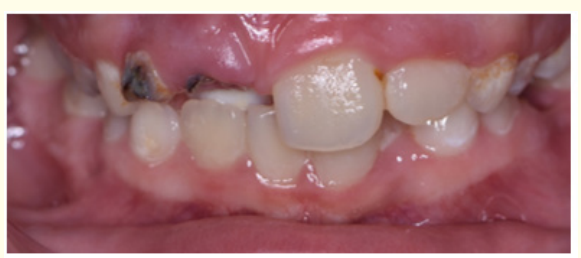

Figure 3a

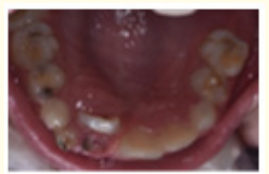

Figure 3b

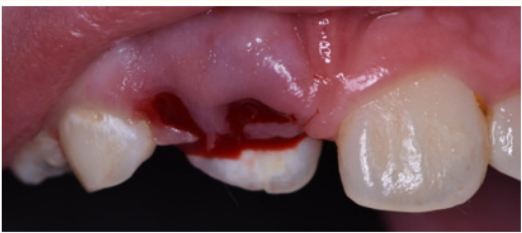

Figure 3c

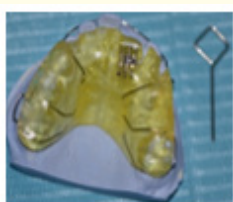

Figure 3d

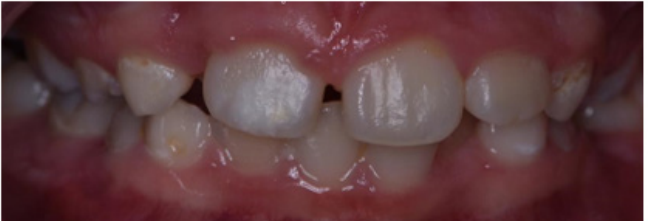

Figure 3e

Figure 3: (a) A 8-year-old male patient with crossbite of 11, 12 teeth numbered. (b) pre-operative occlusal view of the maxillary teeth. (c) after extraction of the persistent primary teeth. (d) The selected removable acrylic appliance with bite plan and screw. (e) Final result after 8 weeks with normal occlusion and position of the maxillary permanent central and lateral incisors. 


\section{Discussion}

Treatment of anterior crossbite is one of the most important concern that guide the child to the normal physiological and psychological development by enhancing the patient's social communications. Such conditions could need only minor interventions to solve the problem in case of early diagnosis. These great results which could be obtained in short periods extremely affect the child's health and social life. Therefore, the clinicians have to know the different methods of managing anterior crossbite to solve any attended child with such highly important conditions seen in their daily work.

In this cases report, the chosen methods of treatment were depending on the advantages and disadvantages of these different methods of the treatment. The first factor affects the decision of using a specific method of treatment was the current status of patient's oral health. The incidence risk of dental caries is high among children during mixed dentition which affected the selected method to keep the home dental care easier by cleaning the appliance and to applicate a perfect home dental care [1]. Fixed appliances were implicated to be cause of many reported complications in previous studies such as plaque accumulation, gingival inflammation and cervical dental caries. Using of short-span fixed wire is one of the methods which represent difficulty of using dental floss and interfere with teeth brushing resulting in more plaque accumulation and high risk of dental caries incidence [8]. One of the methods that help to correct the anterior crossbite with minimum chance of plaque accumulation is Plans Direct Tracks (PDTs) which has to be designed in the laboratory by covering the occlusal surfaces of the primary molars with inclined planes directed from distal to mesial for the anterior crossbite and lingual to buccal for the posterior crossbite [9]. Even though the last mentioned-above advantage of PDTs, it was not selected in our treatment plan because it has to be used only for primary dentation and advised to be used only in early diagnosed conditions to prevent TMJ complications [10]. As the pre-operative figures $(1 \mathrm{a}, 2 \mathrm{a}, 3 \mathrm{a})$ illustrate a bad oral hygiene with persistent defected primary teeth in the third case (Figure 3a), all fixed methods were excluded in this study and the removable acrylic appliance was preferred to avoid incidence of plaque accumulation and gingival inflammation. One of the excluded methods is tongue blade method which needs more cooperation and early diagnosis to move one single tooth. Although that tongue blade is considered simple and economical, it was excluded in this present study due to two reasons; the first reason is the late stage of tooth eruption of the involved teeth in the first two cases (Figure 1a and 2a) and the second reason is the total number of the teeth required to be treated in each case of our cases which needed more intervention by using other methods rather than tongue blade. In addition to that drawback of tongue blade, the amount of its applied force is not accurately measured according to a previous study [11].

The reversed stainless-steel crown is another simple and economical method to treat the anterior crossbite but it was excluded because of the high appearance values of all patients and parents who attended to our dental practice due to esthetical concern.

The accepted esthetic of the composite inclined method needs more time on dental chair and it is costly [12]. It was not preferred because all the patients in this present study were fluctuant cooperative and the procedure of resin material generally is highly sensitive technique which needs patient's cooperation to achieve a proper isolation for successful procedure.

In addition to the above-mentioned methods, fixed orthodontic treatments which lead to a good result in a short time in comparing with the other appliances was also excluded due to many disadvantages such as time-consuming, high cost, risk of plaque accumulation, sensitive technique and incidence of dental caries [8].

Although that the treatment needs shorter time by using the fixed orthodontic treatments, both of the fixed orthodontic methods or the removable acrylic appliances with bite planes will treat the anterior crossbite effectively [13].

The selected method for all cases in this present study is the removable acrylic appliance with bite plane and soldered metal finger springs or screw for the activation. The decision of this selection depended on many advantages of this method which include the ability of clean the appliance regularly, the ability to reach all teeth surface during teeth brushing and maintain a good oral hygiene, no risk of harming the soft tissue, comfortability with using it, simple to be designed, easy to control the amount of the applied forces, easily usable method, can be used with children in early stages and economical [1]. In the third case (Figure 3a), one single tooth was involved and required to be treated. This single tooth was in the first stage of eruption with only one third of the crown was clinically appeared. This patient came from a place located far away from our practice and therefore the selected method to activate the removable appliance was by using a screw due to its simplicity to 
be activated at home. The treatment duration of this case was longer than the other cases because the tooth was partially erupted and needs more time to migrate out the crossbite and to be in normal labial aligned position. This case (Figure 3d) was referred to the orthodontist after our treatment because the crowding which prevented the eruption of the adjacent maxillary permanent lateral incisor and the orthodontist preferred keeping the child under control with regular follow-ups to evaluate the available space periodically in the orthodontic department. Although that the maxillary permanent lateral incisor not yet erupted, the patient and the parent were satisfied with result and this satisfaction will affect directly on the patient's social life in a good matter.

In our study, the maxillary anterior teeth were guided to their normal position by using the removable acrylic appliances with bite plan which were preferred than the other methods to maintain good oral hygiene, to reach the treatment goal with high patient and parents' motivation, to avoid intolerances by using other unacceptable method and finally to enhance the child's dental experience by avoiding any time-consuming or complicated methods.

\section{Conclusion}

Treatment of anterior crossbite by using the removable acrylic appliance with bite plan is advised to be preferred for many advantages. It can be used in early and late developmental stages. The clinician will find this method effective, the parents will be satisfied with the final result and the child will experienced the simplicity of the dental treatment.

\section{Disclosure of Potential Conflicts of Interest}

Three patients were attended in different times in our pediatric dental practice with chief complaint of crowding and esthetically displeasure showed one or two permanent maxillary incisors position behind the permanent lower incisors in each case. Treatments had been done within 6 to 8 weeks according to each case severity. Removable acrylic appliances with bite plan were the used treatment methods in the three cases. The patients and their parents were satisfied after the final results. Pre-operative and postoperative photographs had been taken for each case with signed consents. The cases were documented to present the results of using this treatment methods. The other non-selected methods of the treatment were discussed in this paper with different advantages and disadvantages of each treatment method.
Bibliography

1. Derya Ceyhan., et al. "Taking a Glance at Anterior Crossbite in Children: Case Series". Contemporary Clinical Dentistry 8.4 (2017): 679-682.

2. Ayca Tuba Ulusoy., et al. "Management of anterior dental crossbite with removable appliances". Contemporary Clinical Dentistry 4.2 (2013): 223-226.

3. Keski-Nisula K., et al. "Occurrence of malocclusion and need of orthodontic treatment in early mixed dentition". American Journal of Orthodontics and Dentofacial Orthopedics 124.6 (2003): 631-638.

4. Major PW., et al. "Treatment of anterior cross-bites in the early mixed dentition". Dental Association 58.7 (1992): 574-575, 578-579.

5. Jacobs SG. "Teeth in cross-bite: the role of removable appliances". Australian Dental Journal 34.1 (1989): 20-28.

6. Park JH and Kim TW. "Anterior crossbite correction with a series of clear removable appliances: a case report". Journal of Esthetic and Restorative Dentistry 21.3 (2009): 149-159; discussion 160.

7. Croll TP and Riesenberger RE. "Anterior crossbite correction in the primary dentition using fixed inclined planes. II. Further examples and discussion". Quintessence International 19.1 (1988): 45-51.

8. S Nagarajan., et al. "Interceptive Correction of Anterior Crossbite Using Short-Span Wire-Fixed Orthodontic Appliance: A Report of Three Cases". Case Reports in Dentistry (2018): 4323945 .

9. Ashwin Devasya., et al. "Acrylic Planas Direct Tracks for Anterior Crossbite Correction in Primary Dentition". International Journal of Clinical Pediatric Dentistry 10.4 (2017): 399-403.

10. Ramirez-Yañez. "Planas Direct Tracks for Early Crossbite Correction”. JCO/JUNE (2003).

11. Asher RS., et al. "Anterior dental crossbite correction using a simple fixed appliance: Case report". Pediatric Dentistry 8 (1986): 53-55.

12. Volkan Arikan., et al. "Using Composite Resin Inclined Plane for the Repositioning of a Laterally Luxated Primary Incisor: A Case Report". European Journal of Dentistry 5.1 (2011): 117120. 
13. Wiedel AP., et al. "Stability of anterior crossbite correction: a randomized controlled trial with a 2-year follow-up". Angle Orthodontist 85.2 (2014): 189-195.

\section{Assets from publication with us}

- Prompt Acknowledgement after receiving the article

- Thorough Double blinded peer review

- Rapid Publication

- Issue of Publication Certificate

- High visibility of your Published work

Website: $\underline{w w w . a c t a s c i e n t i f i c . c o m / ~}$

Submit Article: www.actascientific.com/submission.php

Email us: editor@actascientific.com

Contact us: +919182824667 